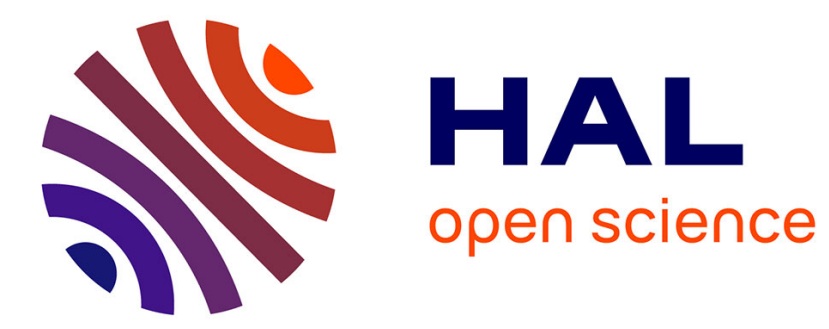

\title{
On least action principles for discrete quantum scales
}

François Dubois, Isabelle Greff, Thomas Hélie

\section{To cite this version:}

François Dubois, Isabelle Greff, Thomas Hélie. On least action principles for discrete quantum scales.

Quantum Interaction, Jun 2012, Paris, France. pp.1-11. hal-01161311

\section{HAL Id: hal-01161311 \\ https://hal.science/hal-01161311}

Submitted on 8 Jun 2015

HAL is a multi-disciplinary open access archive for the deposit and dissemination of scientific research documents, whether they are published or not. The documents may come from teaching and research institutions in France or abroad, or from public or private research centers.
L'archive ouverte pluridisciplinaire HAL, est destinée au dépôt et à la diffusion de documents scientifiques de niveau recherche, publiés ou non, émanant des établissements d'enseignement et de recherche français ou étrangers, des laboratoires publics ou privés. 


\title{
On least action principles for discrete quantum scales
}

\author{
François Dubois ${ }^{a}$, Isabelle Greff ${ }^{b}$ and Thomas Hélie ${ }^{c}$ \\ ${ }^{a}$ Conservatoire National des Arts et Métiers, Paris, France. \\ ${ }^{b}$ Department of Mathematics, University of Pau, France. \\ ${ }^{c}$ IRCAM, Paris, France. \\ francois.dubois@cnam.fr, isabelle.greff@univ-pau.fr, \\ thomas.helie@ircam.fr
}

\begin{abstract}
We consider variational problems where the velocity depends on a scale. After recalling the fundamental principles that lead to classical and quantum mechanics, we study the dynamics obtained by replacing the velocity by some physical observable at a given scale into the expression of the Lagrangian function. Then, discrete Euler-Lagrange and Hamilton-Jacobi equations are derived for a continuous model that incorporates a real-valued discrete velocity. We also examine the paradigm for complex-valued discrete velocity, inspired by the scale relativity of Nottale. We present also rigorous definitions and preliminary results in this direction.
\end{abstract}

Keywords: quantum operators, scale relativity.

\section{Some philosophical principles for Physics}

In this contribution, we first introduce some general philosophical hypotheses that are also widely discussed by several authors (see e.g. Bitbol [1], d'Espagnat [4], Filk and von Müller [5] among others). We set three hypotheses. The two first ones are of ontological type and the third one is concerned with experiments.

(H1)-Principle of reality. It exists a reality which is independent of any observer.

(H2)-Continuous space-time. The space-time is a continuous manifold on which the movement of particles can be described by continuous trajectories.

(H3)-Measurement and scale. The measurement of a physical quantity (time, space, velocity, energy, etc) involves a notion of scale.

- In classical physics, hypothesis (H2) is more constrained: trajectories are supposed to be differentiable or more regular. In this case, the particle velocity is uniquely defined by $v=\frac{\mathrm{d} q}{\mathrm{~d} t}$ which is independent of the scale. Observe that if the trajectory is not regular (continuous but nowhere differentiable) or if some general hypothesis of continuous but non-differentiable space-time is done (as in scale relativity [12]), hypothesis (H3) remains true but the previous velocity has no meaning. On the contrary, a discrete velocity associated with a given scale can still be well-defined. 
- This framework leads to a first paradigm (labelled by the letter "a" in table 1) of continuous classical physics. We recall in section 2 the main point about Euler-Lagrange and Hamilton-Jacobi equations. As noticed by Gondran [7], a complexification of the Hamilton-Jacobi framework provides a natural introduction to the Schrödinger equation. This second paradigm (letter "b" in table 1) is shortly displayed in section 3. As a consequence the differentiability of the trajectories is lost and they can be interpreted in terms of Brownian motion (see e.g. Nelson [11]).

- In this contribution, we develop a scale point of view based on the analysis of reality associated with observations at a given discrete scale. We develop in section 4 a paradigm (labeled with the letter "c" in table 1) based on the knowledge of real-valued discrete velocities. In other words, the velocity at a given scale remains a real number. The idea of introducing discrete operators as fundamental principles of mechanics and quantum mechanics has been proposed by several authors as Greenspan [8], Friedberg and Lee [6] and recently by Khrennikov et al. $[9,10]$ as well as Odake and Sasaki [13]. Nevertheless, our approach does not follow the paradigms suggested by the above references. Our objective is to develop our understanding of the ideas of Nottale [12] who introduced a set of discrete complex velocities (see the label "d" in table 1). We propose some preliminary remarks in this direction in section 5 .

\begin{tabular}{|l|l|l|}
\hline & Continuous Geometry & Given Scale Geometry \\
\hline Classical Physics & (a) Hamilton-Jacobi & (c) Real-valued velocity \\
\hline Quantum Physics & (b) Schrödinger & (d) Complex-valued velocity \\
\hline
\end{tabular}

Table 1. Proposition of four paradigms.

\section{Some classical results on Hamilton-Jacobi equations}

In order to reduce the notations, a Lagrangian function $L(x, v)$ independent of the time is given. To fix the ideas, this Lagrangian can be chosen as

$$
L(x, v)=\frac{1}{2} m v^{2}-\varphi(x) .
$$

The potential energy $\varphi(x)$ structures the space-time with objects governed by physical laws (H1), whereas the kinetic energy $K(v) \equiv \frac{m v^{2}}{2}$ catches the dynamics through the velocity. Consider a regular trajectory $\theta \stackrel{2}{\longmapsto} X(\theta)$ for $0 \leq \theta \leq t$ and the associated action

$$
A(t, X(\bullet))=\int_{0}^{t} L\left(X(\theta), \frac{\mathrm{d}}{\mathrm{d} \theta} X(\theta)\right) \mathrm{d} \theta .
$$

For an arbitrary variation $\delta t$ and for all $C^{1}$-functions $X$ and associated variations $\delta X$, we introduce the variation $\delta A$ of the action. It is given by: 


$$
\begin{aligned}
\delta A(t, & X(\bullet))=L\left(X(t), \frac{\mathrm{d}}{\mathrm{d} \theta} X(t)\right) \delta t \\
& +\int_{0}^{t}\left[\partial_{x} L\left(X(\theta), \frac{\mathrm{d}}{\mathrm{d} \theta} X(\theta)\right) \delta X(\theta)+\partial_{v} L\left(X(\theta), \frac{\mathrm{d}}{\mathrm{d} \theta} X(\theta)\right) \frac{\mathrm{d}}{\mathrm{d} \theta} \delta X(\theta)\right] \mathrm{d} \theta
\end{aligned}
$$

and after integrating by parts:

$$
\begin{gathered}
\delta A=L\left(X(t), \frac{\mathrm{d}}{\mathrm{d} \theta} X(t)\right) \delta t+\int_{0}^{t} \frac{\mathrm{d}}{\mathrm{d} \theta}\left[\partial_{v} L\left(X(\theta), \frac{\mathrm{d}}{\mathrm{d} \theta} X(\theta)\right) \delta X(\theta)\right] \mathrm{d} \theta \\
+\int_{0}^{t}\left(\partial_{x} L\left(X(\theta), \frac{\mathrm{d}}{\mathrm{d} \theta} X(\theta)\right)-\frac{\mathrm{d}}{\mathrm{d} \theta}\left[\partial_{v} L\left(X(\theta), \frac{\mathrm{d}}{\mathrm{d} \theta} X(\theta)\right)\right]\right) \delta X(\theta) \mathrm{d} \theta .
\end{gathered}
$$

- Let $q_{0}$ be fixed and consider the class of functions $\mathcal{C}_{q_{0}}^{1}(0, t)=\left\{X \in \mathcal{C}^{1}(0, t)\right.$ such that $\left.X(0)=q_{0}\right\}$. Notice that the difference between two functions of $\mathcal{C}_{q_{0}}^{1}(0, t)$ belongs to $\mathcal{C}_{0}^{1}(0, t)$. Thus, if $\delta X \in \mathcal{C}_{0}^{1}(0, t)$, then $\delta X(0)=0$. Vanishing the first variations of the action leads to the well-known Euler-Lagrange equation given by

$$
\begin{gathered}
\partial_{x} L\left(X(\theta), \frac{\mathrm{d}}{\mathrm{d} \theta} X(\theta)\right)-\frac{\mathrm{d}}{\mathrm{d} \theta}\left[\partial_{v} L\left(X(\theta), \frac{\mathrm{d}}{\mathrm{d} \theta} X(\theta)\right)\right]=0, \\
\text { with } X(0)=q_{0}, X(t)=q .
\end{gathered}
$$

Moreover, for any arbitrary time $t>0$ and any arbitrary state $q$, let $X^{\text {opt }}(\bullet ; t, q)$ be the solution $X(\bullet)$ in $\mathcal{C}_{q_{0}}^{1}(0, t)$ of the Dirichlet boundary problem given by the Euler-Lagrange equation (3). Observe that $X^{\mathrm{opt}}(\bullet ; t, q)$ is parameterized by the time of arrival $t$ and the value $q$, as precised in (3). At fixed time $t$ and position $q$, the optimal trajectory $X^{\mathrm{opt}}(\bullet ; t, q)$ is supposed to exist and to be unique. We have the initial condition $X^{\mathrm{opt}}(0 ; t, q)=q_{0}$ and the final condition $X^{\mathrm{opt}}(t ; t, q)=q$. Moreover the trajectory $\theta \longmapsto X^{\mathrm{opt}}(\theta ; t, q)$ has a velocity at time $t$ and position $q$ equal to $\left.\partial_{\theta} X^{\mathrm{opt}}(\theta ; t, q)\right|_{\theta=t}$ that can also be considered as a "natural" velocity $\frac{\mathrm{d} q}{\mathrm{~d} t}(t)=\left.\partial_{\theta} X^{\mathrm{opt}}(\theta ; t, q)\right|_{\theta=t} ^{\theta=t}$.

- Let the momentum $p(t, q)$ be defined by

$$
p(t, q)=\partial_{v} L\left(q, \partial_{\theta} X^{\mathrm{opt}}(t ; t, q)\right)
$$

and the optimal action $S(t, q)$ as the action along the optimal trajectory :

$$
S(t, q)=A\left(t, X^{\mathrm{opt}}(\bullet ; t, q)\right) .
$$

At fixed time $t$, due to the Euler-Lagrange equation (3), we deduce from (2) that $\delta A\left(t, X^{\mathrm{opt}}(\bullet)\right)=\partial_{v} L\left(q \partial_{\theta} X^{\mathrm{opt}}(t ; t, q)\right)=p(t, q)$. In other words, the first variation of the optimal action with respect to the final state is the momentum, namely

$$
\partial_{q} S(t, q)=p(t, q) .
$$

If time $t$ is varying and considering the optimal trajectory $\theta \longmapsto X^{\mathrm{opt}}(\theta ; t, q)$, we have $\partial_{t} A\left(t, X^{\mathrm{opt}}(\bullet)\right)=L\left(q, \partial_{\theta} X^{\mathrm{opt}}(t ; t, q)\right)$. Wrinting that this quantity is 
the time variation of the optimal action (5) and taking into account the velocity of the optimal trajectory at the location $q$, we deduce

$$
\partial_{t} S+\partial_{q} S \bullet \partial_{\theta} X^{\mathrm{opt}}(t ; t, q)=\partial_{t} A\left(t, X^{\mathrm{opt}}(\bullet)\right)=L\left(q, \partial_{\theta} X^{\mathrm{opt}}(t ; t, q)\right) .
$$

- Introduce now the Legendre transform of the Lagrangian $L$ relatively to the second variable $v$. Suppose that the function $v \longmapsto y=\partial_{v} L(x, v)$ is invertible and denote by $V(x, y)$ its inverse. The Hamiltonian $H(y, x)$ is classically defined by

$$
H(y, x)=y \bullet V(x, y)-L(x, V(x, y)) .
$$

Observe that if (4) holds then $\partial_{\theta} X^{\mathrm{opt}}(t ; t, q)=V(q, p)$ and $H(p, q)=p \bullet V(q, p)$ $-L(q, V(q, p))$. We deduce from $(7)$,

$$
\begin{aligned}
L\left(q, \partial_{\theta} X^{\mathrm{opt}}(t ; t, q)\right) & =\partial_{t} S+\left(\partial_{q} S\right) \bullet \partial_{\theta} X^{\mathrm{opt}}(t ; t, q) \\
& =\partial_{t} S+p(t, q) \bullet V(q, p(t, q)) .
\end{aligned}
$$

This leads to the well-known Hamilton-Jacobi equation

$$
\partial_{t} S+H\left(\partial_{q} S, q\right)=0 .
$$

\section{How to derive the Schrödinger equation ?}

The "break through" from classical Hamilton-Jacobi equations to quantum dynamics is due to Schrödinger [14]. Introduce the wave function $\psi$ according to

$$
\psi=\exp \left(i \frac{S}{\hbar}\right)
$$

and inject this relation into (4) and (8). We get $\frac{i}{\hbar} \mathrm{d} S=\frac{1}{\psi} \mathrm{d} \psi$ and due to (6), we have $p=\frac{\hbar}{i} \frac{1}{\psi} \partial_{q} \psi$. Then Schrödinger transforms the momentum $p$ into the so-called momentum operator $P$ defined by $P \bullet \psi \equiv-i \hbar \partial_{q} \psi$. Observe that the momentum $P$ becomes now a complex derivative operator. Starting from the usual Lagrangian, we observe that the good generalisation of quantum mechanic of $v^{2}$ is not $|v|^{2}$ (or $P P^{*}$ ) but $v v$ (or $P P$ in the classical formalism). Then the Hamiltonian $H$ takes the expression $H=\frac{1}{2 m} P^{2}+\varphi(q)=-\frac{\hbar^{2}}{2 m} \Delta+\varphi(q)$ and the Schrödinger equation

$$
i \hbar \partial_{t} \psi=-\frac{\hbar^{2}}{2 m} \Delta \psi+\varphi(q) \psi
$$

is a direct consequence of the Hamilton-Jacobi equation (8).

- An other way to derive the Schrödinger equation has been proposed by Nottale [12]. The idea consists in replacing the classical trajectory derivative $\frac{\mathrm{d}}{\mathrm{d} t} \equiv \partial_{t}+v \bullet \partial_{q}$ by the complex Dynkin operator $\frac{\mathrm{d}}{\mathrm{d} t} \equiv \partial_{t}+v \bullet \partial_{q}-i \frac{\hbar}{2 m} \Delta$. Then, equation (7) takes the form: $L=\partial_{t} S+v \bullet\left(\partial_{q} S\right)-i \frac{\hbar}{2 m} \Delta S$ and $\partial_{t} S+\frac{1}{m}\left(\partial_{q} S\right)^{2}-i \frac{\hbar}{2 m} \Delta S+\varphi(q)-\frac{m}{2}\left(\frac{1}{m} \partial_{q} S\right)^{2}=0$. Following Gondran [7], one can derive a complex Hamilton-Jacobi equation

$$
\partial_{t} S+\frac{1}{2 m}\left(\partial_{q} S\right)^{2}+\varphi(q)-i \frac{\hbar}{2 m} \Delta S=0 .
$$


If we decompose the complex optimal action $S$ into its real and imaginary parts, $i d$ est $S=\Sigma-i \hbar \log R$, an elementary calculus allows to transform the complex Hamilton-Jacobi equation (11) into the form proposed by Bohm and Hiley [2]:

$$
\partial_{t} \Sigma+\frac{1}{2 m}\left(\partial_{q} \Sigma\right)^{2}+\varphi(q)-\frac{\hbar}{2 m} \frac{\partial_{q} R}{R}=0, \quad \partial_{t} R^{2}+\operatorname{div}\left(\frac{R^{2}}{m} \partial_{q} \Sigma\right)=0 .
$$

The quantum potential $Q \equiv-\frac{\hbar}{2 m} \frac{\partial_{q} R}{R}$ is the quantity that has to be added to transform the classical Hamilton-Jacobi equation (8) into the real part of the complex Hamilton-Jacobi equation (11).

- Introduce now the change of variables (9) into the complex Hamilton-Jacobi equation (11). If we derive once again the relation $\frac{i}{\hbar} \partial_{q} S=\frac{1}{\psi} \partial_{q} \psi$ towards the space variable $q$, we get $\frac{i}{\hbar} \partial_{q}^{2} S=-\frac{1}{\psi^{2}}\left(\partial_{q} \psi\right)^{2}+\frac{1}{\psi} \partial_{q}^{2} \psi$. The left hand side of the complex Hamilton-Jacobi equation (11) is now equal to

$$
\frac{1}{\psi}\left[\frac{\hbar}{i} \partial_{t} \psi+\frac{1}{2 m \psi}\left(\frac{\hbar}{i} \partial_{q} \psi\right)^{2}+\varphi(q) \psi-i \frac{\hbar}{2 m} \frac{\hbar}{i}\left(-\frac{1}{\psi}\left(\partial_{q} \psi\right)^{2}+\partial_{q}^{2} \psi\right)\right]
$$
and the Schrödinger equation (10) is established.

\section{Real-valued discrete-measured velocity at a given scale}

We consider now that the classical velocity is not a relevant observable. We introduce a given strictly positive scale parameter $\varepsilon$, a "fat" initial condition $q_{0} \in \mathcal{C}([-\varepsilon, 0])$ as a continuous function and the classical discrete so-called finite difference operators

$$
\left(\mathrm{d}_{\varepsilon}^{-} q\right)(\theta) \equiv \frac{1}{\varepsilon}(q(\theta)-q(\theta-\varepsilon)), \quad\left(\mathrm{d}_{\varepsilon}^{+} q\right)(\theta) \equiv \frac{1}{\varepsilon}(q(\theta+\varepsilon)-q(\theta)) .
$$

Let us notice that the velocity $v_{\varepsilon}^{ \pm}=\mathrm{d}_{\varepsilon}^{ \pm} q$ is now measured at the given scale $\varepsilon$ by two possible schemes (13), as a consequence of the hypothesis (H3). We consider a given (final) time $t$ strictly positive and a continuous trajectory $([-\varepsilon, t] \ni \theta \longmapsto q(\theta)) \in \mathcal{C}([-\varepsilon, t])$ with the initial condition $q_{0}$. This initial condition is not classical, $q_{0}$ is not anymore given at a time $t=0$, but on a small interval depending on the scale $\varepsilon$. It has to be considered in the following sense: restricted to the interval $[-\varepsilon, 0]$, function $q$ is equal to the given function $q_{0}$. As in the classical case described in section 2 , we introduce an action $A$ based on a regular Lagrangian $L(x, v)$ which is similar to that introduced at the relation (1):

$$
A(t, q) \equiv \int_{0}^{t} L\left(q(\theta), \mathrm{d}_{\varepsilon}^{-} q(\theta)\right) \mathrm{d} \theta .
$$

In the following, we examine the choice of $\mathrm{d}_{\varepsilon}^{-} q$ as the observed velocity. Thus the paradigm based on this choice and (14) is studied. We have just formally replaced velocity $v$ in the second argument of the Lagrangian (1) by the discrete velocity $v_{\varepsilon}=\mathrm{d}_{\varepsilon}^{-} q$. We have the following result. 
- Proposition 1. Variation of the discrete action

The variation $\delta A$ of the action $A$ defined in (14) when trajectory $q$ is varying by an increment $\delta q$ and time by an increment $\delta t$ is given by

$$
\begin{gathered}
\delta A=L \delta t-\frac{1}{\varepsilon} \int_{-\varepsilon}^{0} \partial_{v} L(\theta+\varepsilon) \delta q(\theta) \mathrm{d} \theta \\
+\int_{t-\varepsilon}^{t}\left[\partial_{x} L+\frac{1}{\varepsilon} \partial_{v} L\right](\theta) \delta q(\theta) \mathrm{d} \theta \\
+\int_{0}^{t-\varepsilon}\left[\partial_{x} L-\mathrm{d}_{\varepsilon}^{+}\left(\partial_{v} L\right)\right](\theta) \delta q(\theta) \mathrm{d} \theta .
\end{gathered}
$$

The first integral in (15) is null a priori since initial condition $q_{0}$ is supposed to be fixed between $-\varepsilon$ and 0 .

Proof of Proposition 1.

Since Lagrangian $L$ in (1) is a regular function, differentiating (14) yields

$$
\begin{aligned}
& \delta A=L \delta t+\int_{0}^{t}\left(\partial_{x} L\right) \delta q(\theta) \mathrm{d} \theta+\int_{0}^{t}\left(\partial_{v} L\right) \frac{1}{\varepsilon}(\delta q(\theta)-\delta q(\theta-\varepsilon)) \mathrm{d} \theta \\
& =L \delta t+\int_{0}^{t}\left(\partial_{x} L\right) \delta q(\theta) \mathrm{d} \theta+\frac{1}{\varepsilon} \int_{0}^{t}\left(\partial_{v} L\right) \delta q(\theta) \mathrm{d} \theta-\frac{1}{\varepsilon} \int_{-\varepsilon}^{t-\varepsilon}\left(\partial_{v} L\right)(\theta+\varepsilon) \delta q(\theta) \mathrm{d} \theta \\
& =L \delta t-\frac{1}{\varepsilon} \int_{-\varepsilon}^{0}\left(\partial_{v} L\right)(\theta+\varepsilon) \delta q(\theta) \mathrm{d} \theta \\
& \quad+\int_{0}^{t-\varepsilon}\left(\partial_{x} L-\frac{1}{\varepsilon}\left[\left(\partial_{v} L\right)(\theta+\varepsilon)-\left(\partial_{v} L\right)(\theta)\right]\right) \delta q(\theta) \mathrm{d} \theta \\
& \quad+\int_{t-\varepsilon}^{t}\left[\partial_{x} L+\frac{1}{\varepsilon} \partial_{v} L\right](\theta) \delta q(\theta) \mathrm{d} \theta, \quad \text { so that (15) is a conse- }
\end{aligned}
$$

quence of the definition of the operator $\mathrm{d}_{\varepsilon}^{+}$given by (13).

- We deduce from relation (15) that an optimal trajectory satisfies the discrete version of the Euler-Lagrange equation, that is

$$
\partial_{x} L\left(q(\theta), \mathrm{d}_{\varepsilon}^{-} q\right)-\mathrm{d}_{\varepsilon}^{+}\left[\partial_{v} L\left(q(\theta), \mathrm{d}_{\varepsilon}^{-} q\right)\right]=0, \quad 0 \leq \theta \leq t-\varepsilon .
$$

This discrete-time dynamics is formally very similar to the classical Euler-Lagrange dynamics (3). Remark that it is nothing but an implicit finite difference scheme:

$$
\begin{gathered}
\partial_{x} L\left(q(\theta), \frac{1}{\varepsilon}(q(\theta)-q(\theta-\varepsilon))\right)-\frac{1}{\varepsilon} \partial_{v} L\left(q(\theta+\varepsilon), \frac{1}{\varepsilon}(q(\theta+\varepsilon)-q(\theta))\right) \\
+\frac{1}{\varepsilon} \partial_{v} L\left(q(\theta), \frac{1}{\varepsilon}(q(\theta)-q(\theta-\varepsilon))\right)=0 .
\end{gathered}
$$

From (17), it is clear that the dynamics of the optimal trajectory is that of a delay system, and more precisely,

$$
q(\theta) \text { is a function of } \theta, q(\theta-\varepsilon), q(\theta-2 \varepsilon) .
$$

Function $q$ is the solution of the two-step finite-difference scheme (17). Because $q_{0}(\theta)$ is known for $-\varepsilon \leq \theta \leq 0$, the knowledge of $q(\theta)$ for $0 \leq \theta \leq \varepsilon$ is generically sufficient for solving the scheme (17) under the form (18). The knowledge 
of $q_{0}$ on $[-\varepsilon, \varepsilon]$ is equivalent to the knowledge of the discrete derivative $\mathrm{d}_{\varepsilon}^{+} q(\theta)$ for $-\varepsilon \leq \theta \leq 0$. Let us define this initial variation $\left(\mathrm{d}_{\varepsilon}^{+} q\right)_{0}$ as

$$
\left(\mathrm{d}_{\varepsilon}^{+} q\right)_{0}(\theta)=\frac{1}{\varepsilon}(q(\theta+\varepsilon)-q(\theta)), \quad-\varepsilon \leq \theta \leq 0 .
$$

- From the knowledge of $q_{0}(\theta)$ and $\left(\mathrm{d}_{\varepsilon}^{+} q\right)_{0}$ we construct a priori without major difficulty the continuous trajectory $q$ solution of (17) of the type (18) for $0 \leq \theta \leq t$. We obtain in this way a "final state" $q^{\mathrm{f}}$ which is now a piece of trajectory $q$ :

$$
q^{\mathrm{f}}(\theta)=q(t+\theta), \quad-\varepsilon \leq \theta \leq 0 .
$$

This leads to the functional $Q_{t}:\left(\mathrm{d}_{\varepsilon}^{+} q\right)_{0} \longmapsto q^{\mathrm{f}}=Q_{t}\left(\left(\mathrm{~d}_{\varepsilon}^{+} q\right)_{0}\right)$ defined from $\mathcal{C}([-\varepsilon, 0])$ to $\mathcal{C}([-\varepsilon, 0])$, for $q_{0}$ fixed. We suppose this functional to be one to one. In consequence, we can suppose the optimal trajectory parameterized by the final state $q^{\mathrm{f}} \in \mathcal{C}([-\varepsilon, 0])$. We denote by $S\left(t, q^{\mathrm{f}}\right)$ the corresponding optimal action. We observe that at fixed $q_{0}$, it depends only on the final time $t$ and the final state $q^{\mathrm{f}}$ whereas the action $A$ is a functional of all the states along the whole trajectory.

\section{- Proposition 2. Derivative of the optimal action}

Under a variation $\delta q^{\mathrm{f}}$ of the final state, the optimal action admits a variation $\delta S\left(t, q^{\mathrm{f}}\right)$ given by

$$
\delta S\left(t, q^{\mathrm{f}}\right) \equiv \frac{\partial S}{\partial q^{\mathrm{f}}} \bullet \delta q^{\mathrm{f}}=\int_{t-\varepsilon}^{t}\left[\partial_{x} L+\frac{1}{\varepsilon} \partial_{v} L\right]\left(q(\theta),\left(\mathrm{d}_{\varepsilon}^{-} q\right)(\theta)\right) \delta q(\theta) \mathrm{d} \theta .
$$

\section{Proof of Proposition 2.}

Due to the discrete Euler-Lagrange equations (16), the optimal trajectory vanishes the third term of the right hand side of the relation (15). The first one is identically null because the initial condition $q_{0}$ remains fixed. The result is then a simple consequence of the relation (15) when time $t$ is fixed.

- In the right hand side of relation (20) the final state is not explicit. In order to exhibit the variation $\delta q^{\mathrm{f}}$ we introduce

$$
\Gamma\left(t, q^{\mathrm{f}}\right)(\theta) \equiv\left(\partial_{v} L+\varepsilon \partial_{x} L\right)\left(q(t+\theta),\left(\mathrm{d}_{\varepsilon}^{-} q\right)(t+\theta)\right), \quad-\varepsilon \leq \theta \leq 0 .
$$

Then, $\Gamma\left(t, q^{\mathrm{f}}\right) \in \mathcal{C}([-\varepsilon, 0])$ and relation $(20)$ can be also written as

$$
\frac{\partial S}{\partial q^{\mathrm{f}}} \bullet \delta q^{\mathrm{f}}=\frac{1}{\varepsilon} \int_{-\varepsilon}^{0} \Gamma\left(t, q^{\mathrm{f}}\right)(\theta) \delta q^{\mathrm{f}}(\theta) \mathrm{d} \theta .
$$

Let us observe that expression $\Gamma\left(t, q^{\mathrm{f}}\right)$ is a good candidate for a momentum variable analogous to the one that satisfies the relation (6) in differentiable mechanics.

- The natural question is now to determinate the "total variation" with time of the optimal action, id est the discrete analogous of the expression (7). This 
is not possible if we restrict to solely continuous trajectories. Nevertheless we propose a result for a discrete variation in time of amplitude exactly equal to $\varepsilon$. We denote by $\widetilde{q}^{\mathrm{f}}$ the trajectory obtained from the final state $q^{\mathrm{f}}$ after a time extension of amplitude $\varepsilon$ : $\widetilde{q}^{\mathrm{f}}(\theta) \equiv q(t+\varepsilon+\theta)$ for $-\varepsilon \leq \theta \leq 0$. Then we have a simple expression for the difference $S\left(t+\varepsilon, \widetilde{q}^{\mathrm{f}}\right)-S\left(t, q^{\mathrm{f}}\right)$ because the two integrals in (14) operates on the same optimal trajectory:

$$
S\left(t+\varepsilon, \widetilde{q}^{\mathrm{f}}\right)-S\left(t, q^{\mathrm{f}}\right)=\int_{t}^{t+\varepsilon} L\left(q(\theta),\left(\mathrm{d}_{\varepsilon}^{-} q\right)(\theta)\right) \mathrm{d} \theta .
$$

- Proposition 3. Discrete variation of the optimal action

Let $\xi$ be a continuous function in the space $\mathcal{C}([-\varepsilon, 0])$. We have

$$
S\left(t, q^{\mathrm{f}}+\xi\right)-S\left(t, q^{\mathrm{f}}\right)=\frac{1}{\varepsilon} \int_{-\varepsilon}^{0}\left[\int_{0}^{1} \Gamma\left(t, q^{\mathrm{f}}+\eta \xi\right)(\theta) \mathrm{d} \eta\right] \xi(\theta) \mathrm{d} \theta .
$$

Proof of Proposition 3.

We introduce $\Phi(\eta) \equiv S\left(t, q^{\mathrm{f}}+\eta \xi\right)$ for $0 \leq \eta \leq 1$. It is a derivable function of the real variable $\eta$ and we have

$$
\begin{aligned}
\frac{\mathrm{d} \Phi}{\mathrm{d} \eta} & =\frac{\partial S}{\partial q^{\mathrm{f}}}\left(t, q^{\mathrm{f}}+\eta \xi\right) \cdot \frac{\mathrm{d}}{\mathrm{d} \eta}\left(q^{\mathrm{f}}+\eta \xi\right)=\frac{\partial S}{\partial q^{\mathrm{f}}}\left(t, q^{\mathrm{f}}+\eta \xi\right) \bullet \xi \\
& =\frac{1}{\varepsilon} \int_{-\varepsilon}^{0} \Gamma\left(t, q^{\mathrm{f}}+\eta \xi\right)(\theta) \xi(\theta) \mathrm{d} \theta .
\end{aligned}
$$

Then the relation (24) is obtained by integration relative to $\eta \in[0,1]$ and using Fubini theorem.

Then, we present here the main result of this contribution.

- Proposition 4. Discrete temporal variation of the optimal action

Let $\Gamma_{\varepsilon}\left(t, q^{\mathrm{f}}\right)$ be a mean value at final time $t$ of the momentum introduced in $(21)$ :

$$
\Gamma_{\varepsilon}\left(t, q^{\mathrm{f}}\right)(\theta) \equiv \int_{0}^{1} \Gamma\left(t+\varepsilon, q^{\mathrm{f}}+\varepsilon \eta\left(\mathrm{d}_{\varepsilon}^{-} q\right)(t+\varepsilon+\theta)\right)(\theta) \mathrm{d} \eta, \quad-\varepsilon \leq \theta \leq 0 .
$$

The following discrete Hamilton-Jacobi type equation holds

$$
\begin{aligned}
\mathrm{d}_{\varepsilon}^{+} S & \left.+\frac{1}{\varepsilon} \int_{-\varepsilon}^{0} \Gamma_{\varepsilon}\left(t, q^{\mathrm{f}}\right)(\theta)\left(\mathrm{d}_{\varepsilon}^{-} q\right)(t+\varepsilon+\theta)\right) \mathrm{d} \theta \\
& -\frac{1}{\varepsilon} \int_{t}^{t+\varepsilon} L\left(q(\tau),\left(\mathrm{d}_{\varepsilon}^{-} q\right)(\tau)\right) \mathrm{d} \tau=0
\end{aligned}
$$

\section{Proof of Proposition 4.}

We recall that $\mathrm{d}_{\varepsilon}^{+} S \equiv \frac{1}{\varepsilon}\left(S\left(t+\varepsilon, q^{\mathrm{f}}\right)-S\left(t, q^{\mathrm{f}}\right)\right)$. Then we have the decomposition

$$
\varepsilon \mathrm{d}_{\varepsilon}^{+} S=-\left(S\left(t+\varepsilon, \widetilde{q}^{\mathrm{f}}\right)-S\left(t+\varepsilon, q^{\mathrm{f}}\right)\right)+\left(S\left(t+\varepsilon, \widetilde{q}^{\mathrm{f}}\right)-S\left(t, q^{\mathrm{f}}\right)\right) .
$$


We remark also that $\widetilde{q}^{\mathrm{f}}(\theta)-q^{\mathrm{f}}(\theta)=q(t+\varepsilon+\theta)-q(t+\theta)=\varepsilon\left(\mathrm{d}_{\varepsilon}^{-} q\right)(t+\varepsilon+\theta)$. Then we have from $(24)$ with $\xi=\epsilon\left(\mathrm{d}_{\varepsilon}^{-} q\right)(t+\varepsilon+\theta)$ :

$$
\begin{aligned}
S(t+\varepsilon & \left., \widetilde{q}^{\mathrm{f}}\right)-S\left(t+\varepsilon, q^{\mathrm{f}}\right)= \\
& =\frac{1}{\varepsilon} \int_{-\varepsilon}^{0}\left[\int_{0}^{1} \Gamma\left(t+\varepsilon, q^{\mathrm{f}}+\varepsilon \eta\left(\mathrm{d}_{\varepsilon}^{-} q\right)(t+\varepsilon+\theta)\right)(\theta) \mathrm{d} \eta\right]\left(\widehat{q}^{\mathrm{f}}(\theta)-q^{\mathrm{f}}(\theta)\right) \mathrm{d} \theta \\
& =\frac{1}{\varepsilon} \int_{-\varepsilon}^{0}\left[\int_{0}^{1} \Gamma\left(t+\varepsilon, q^{\mathrm{f}}+\varepsilon \eta\left(\mathrm{d}_{\varepsilon}^{-} q\right)(t+\varepsilon+\theta)\right)(\theta) \mathrm{d} \eta\right] \varepsilon\left(\mathrm{d}_{\varepsilon}^{-} q\right)(t+\varepsilon+\theta) \mathrm{d} \theta
\end{aligned}
$$

and the second term of the left hand side of the relation (26) is clear. The end of the proof is a consequence of the decomposition of $\varepsilon \mathrm{d}_{\varepsilon}^{+} S$ and relation (23).

- The analogy between the classical Hamilton-Jacobi equation (8) and the discrete version (26) is clear. We observe that the Lagrangian is replaced by its mean value on an interval of size $\varepsilon$. Moreover the natural associated momentum $\Gamma_{\varepsilon}\left(t, q^{\mathrm{f}}\right)(\theta)$ defined at relation (25) is not a priori strictly equal to the momentum $\Gamma\left(t, q^{\mathrm{f}}\right)(\theta)$ introduced at relation (21). This splitting at the discrete scale of the moment $p$ satisfying both relations (4) and (6) is a real difficulty that we will consider in a future contribution.

\section{Towards complex-valued discrete-measured velocity}

The discrete scaled velocity $v_{\varepsilon}=\mathrm{d}_{\varepsilon}^{-} q$ introduced in section 4 is purely real. We consider now a complex discrete velocity $v_{\varepsilon}$. Following an idea proposed by Nottale [12], we introduce a discrete complex derivation operator $\square_{\varepsilon}$ according to

$$
\left(\square_{\varepsilon} q\right)(\theta) \equiv \frac{1}{2 \varepsilon}(q(\theta+\varepsilon)-q(\theta-\varepsilon))+\frac{i \mu}{2 \varepsilon}(q(\theta+\varepsilon)-2 q(\theta)+q(\theta-\varepsilon)),
$$

with $\mu^{2}=1$. We decompose the discrete operator $\square_{\varepsilon} q$ under the form $\square_{\varepsilon} q \equiv \square_{\varepsilon}^{\mathrm{r}} q+i \mu \square_{\varepsilon}^{\mathrm{i}} q$. We have

$$
\begin{aligned}
& \left(\square_{\varepsilon}^{\mathrm{r}} q\right)(\theta) \equiv \frac{1}{2 \varepsilon}(q(\theta+\varepsilon)-q(\theta-\varepsilon))=\frac{1}{2}\left(\mathrm{~d}_{\varepsilon}^{+} q(\theta)+\mathrm{d}_{\varepsilon}^{-} q(\theta)\right) \\
& \left(\square_{\varepsilon}^{\mathrm{i}} q\right)(\theta) \equiv \frac{1}{2 \varepsilon}(q(\theta+\varepsilon)-2 q(\theta)+q(\theta-\varepsilon))=\frac{1}{2}\left(\mathrm{~d}_{\varepsilon}^{+} q(\theta)-\mathrm{d}_{\varepsilon}^{-} q(\theta)\right) .
\end{aligned}
$$

The real part $\square_{\varepsilon}^{\mathrm{r}} q$ is the standard time derivative for regular trajectories when $\varepsilon$ goes to 0 . The imaginary part $\square_{\varepsilon}^{\mathrm{i}} q$ is asymptotically null for a regular function and accounts for the slope jump at a given time. This framework has been proven to be well-posed by Cresson and Greff [3] introducing a limit when $\varepsilon$ goes to zero in a well-defined projection functional space.

- As remarked previously, the appropriate generalization of the kinetic energy $\frac{m}{2} v^{2}$ is obtained by taking the (complex) square of the momentum operator. So in the expression of the Lagrangian we have to replace $v^{2}$ by $\left(\square_{\varepsilon} q\right)^{2}$. We set

$$
K_{\varepsilon} \equiv \frac{m}{2}\left(\square_{\varepsilon} q\right)^{2}=\frac{m}{2}\left[\left(\square_{\varepsilon}^{\mathrm{r}} q\right)^{2}-\left(\square_{\varepsilon}^{\mathrm{i}} q\right)^{2}+2 i \mu\left(\square_{\varepsilon}^{\mathrm{r}} q\right)\left(\square_{\varepsilon}^{\mathrm{i}} q\right)\right] .
$$


If $K_{\varepsilon}$ is real, i.e. $\operatorname{Im} K_{\varepsilon}=0$, the product $\left(\square_{\varepsilon}^{\mathrm{r}} q\right)\left(\square_{\varepsilon}^{\mathrm{i}} q\right)$ is null and two cases occur.

(i) If $K_{\varepsilon} \geq 0$, then $\square_{\varepsilon}^{\mathrm{i}} q=0$ and we have a natural reference to a regular trajectory.

(ii) If $K_{\varepsilon}<0$, then then $\square_{\varepsilon}^{\mathrm{r}} q=0$. The position $q(\theta)$ is essentially unchanged during one $\varepsilon$-step but the jump is not null and the direction of the trajectory has changed abruptly.

If the kinetic energy is imaginary, $\operatorname{Re} K_{\varepsilon}=0$ and we have $\left(\square_{\varepsilon}^{\mathrm{r}} q\right)^{2}=\left(\square_{\varepsilon}^{\mathrm{i}} q\right)^{2}$ that implies $\mathrm{d}_{\varepsilon}^{+} q(\theta)=0$ or $\mathrm{d}_{\varepsilon}^{-} q(\theta)=0$. The particle has not moved just before time $t$ or just after!

- We consider now the iterate of the operator $\square_{\varepsilon}$ with itself. This type of algebraic formula is natural for the extension of $\frac{\mathrm{d}}{\mathrm{d} t}\left(m \frac{\mathrm{d}}{\mathrm{d} t}\right)$ in the Euler-Lagrange equation. We emphasise the role of $\mu^{2}=1$ when we consider the composed operator. We have

$$
\left\{\begin{array}{l}
\operatorname{Re}\left(\left(\square_{\varepsilon} \circ \square_{\varepsilon}\right) q\right)=\left(1-\mu^{2}\right)\left(\square_{\varepsilon}^{\mathrm{r}} \circ \square_{\varepsilon}^{\mathrm{r}}\right) q+\mu^{2}\left(\square_{\frac{\varepsilon}{2}}^{\mathrm{r}} \circ \square_{\frac{\varepsilon}{2}}^{\mathrm{r}}\right) q \\
\operatorname{Im}\left(\left(\square_{\varepsilon} \circ \square_{\varepsilon}\right) q\right)=2 \mu\left(\square_{\varepsilon}^{\mathrm{r}} \circ \square_{\varepsilon}^{\mathrm{i}}\right) q .
\end{array}\right.
$$

Roughly speaking the product "jump by jump" allows to recover some regularity at a smaller scale $\varepsilon / 2$.

- We propose to introduce the following complex action for $q \in \mathcal{C}([-\varepsilon, t+\varepsilon])$ :

$$
A_{\varepsilon}(t, q) \equiv \int_{0}^{t} L\left(q(\theta),\left(\square_{\varepsilon} q\right)(\theta)\right) \mathrm{d} \theta=\int_{0}^{t}\left[\frac{m}{2}\left(\square_{\varepsilon} q\right)^{2}-\varphi(q(\theta))\right] \mathrm{d} \theta .
$$

Our working plan follows the ideas presented in sections 2 and 3. In an analogous way as the one proposed in section 3, we will consider the Euler-Lagrange optimality condition, introduce the optimal trajectories, derive a Hamilton-Jacobi like equation for the optimal value of the action. Then make the change of variable (9) to transform the evolution equation (26) into a Schrödinger type equation.

\section{References}

1. M. Bitbol. Mécanique quantique, une introduction philosophique, ChampsFlammarion, Paris, 1997.

2. D. Bohm, B.J. Hiley. The Undivided Universe: An Ontological Interpretation of Quantum Theory, Routledge, New York,1993.

3. J. Cresson, I. Greff. "Non-differentiable embedding of Lagrangian systems and partial differential equations", Journal of Mathematical Analysis and Applications, vol. 384, issue 2, p. 626-646, 2011.

4. B. d'Espagnat. Le réel voilé ; Analyse des concepts quantiques, Fayard, Paris, 1994.

5. T. Filk, A. von Müller. "Quantum physics and consciousness: The quest for a common conceptual foundation", Mind and Matter, vol. 7, p. 59-79, 2009.

6. R. Friedberg, T.D. Lee. Discrete Quantum Mechanics, Nuclear Physics B., vol. 225, issue 1, p. 1-52, october 1983. 
7. M. Gondran. "Complex calculus of variations and explicit solutions for complex Hamilton-Jacobi equations", C. R. Acad. Sci. Paris, vol. 332, Série I, p. 677-680, 2001. "Complex analytical mechanics, complex nonstandard stochastic process and quantum mechanics", C. R. Acad. Sci. Paris, vol. 333, Série I, p. 593-598, 2001.

8. D. Greenspan. A new explicit discrete mechanics with applications, J. Franklin Institute, vol. 294, p. 231-240, 1972.

9. A. Khrennikov, Ja. I. Volovich. "Discrete time dynamical models and their quantum-like context-dependent properties", J. Modern Optics, vol. 51, issue 6/7, p. 113-114, 2004.

10. A. Khrennikov. "Discrete time dynamics", Contextual approach to quantum formalism, chapter 12, Springer, Berlin-Heidelberg-New York, 2009.

11. E. Nelson. "Derivation of the Schrödinger Equation from Newtonian Mechanics", Physical Review, vol. 150, p. 1079-1085, 1966.

12. L. Nottale. Fractal space-time and microphysics: towards a theory of scale relativity, 333 p., World Scientific, 1993.

13. S. Odake, R. Sasaki. "Discrete Quantum Mechanics", Journal of Physics A: Mathematical and Theoretical, vol. 44, issue 35, 353001, 2011.

14. E. Schrödinger. "Quantizierung als Eigenwertproblem (Erste Mitteilung), Annalen der Physik, vol. 79, p. 361-376, "Über das Verhältnis der Heisenberg Born Jordanischen Quantenmechanik zu der meinen", Annalen der Physik, vol. 79, p. 734-756, 1926. 